\title{
eJRIEPS
}

Ejournal de la recherche sur l'intervention en éducation physique et sport

$18 \mid 2009$

Varia

\section{Rôle du contexte dans la formation d'élèves au tutorat réciproque en tennis de table}

Pierre Ensergueix et Lucile Lafont

\section{(2) OpenEdition}

Journals

Édition électronique

URL : http://journals.openedition.org/ejrieps/5498

DOI : 10.4000 /ejrieps. 5498

ISSN : 2105-0821

Éditeur

ELLIADD

Référence électronique

Pierre Ensergueix et Lucile Lafont, «Rôle du contexte dans la formation d'élèves au tutorat réciproque en tennis de table », eJRIEPS [En ligne], 18| 2009, mis en ligne le 01 juillet 2009, consulté le 18 mars 2021. URL : http://journals.openedition.org/ejrieps/5498; DOI : https://doi.org/10.4000/ejrieps.5498

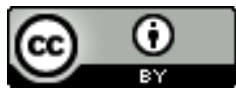

La revue eJRIEPS est mise à disposition selon les termes de la Creative Commons Attribution 4.0 International License. 
Rôle du contexte dans la formation d'élèves au tutorat réciproque en tennis de table

Pierre Ensergueix \& Lucile Lafont

Université Bordeaux 2, France.

\section{Résumé}

Cet article s'inscrit dans un programme de recherche (Équipe VSTII, Laboratoire LACES 4140, Université Bordeaux 2, France) visant l'identification des conditions favorables à la mise en œuvre de procédures de tutorat entre pairs en Education Physique et Sportive (EPS). À ce jour, les résultats des études de ce programme ont montré que la formation d'élèves tuteurs à l'exercice de leur fonction engendrait des bénéfices à la fois chez les tutorés (Lafont, Cicéro, Vedel, \& Viala, 2005) et chez les tuteurs eux-mêmes (Cicéro \& Lafont, 2007). La nécessité de préparer les élèves au managérat réciproque en tennis de table dans l'attente raisonnée de progrès moteurs a également été démontrée (Ensergueix \& Lafont, 2007). Toutefois, ces formations ont été uniquement conduites en contexte expérimental, limitant de fait leur validité en contexte écologique de classe entière. L'objectif de cette présentation est de mettre en regard l'usage du tutorat réciproque en contexte expérimental et en contexte de classe entière du point de vue de la formation des élèves tuteurs. Proposer des dispositifs de formation adaptés à chacun des contextes suppose de surmonter un large faisceau de contraintes dont certaines sont vraiment spécifiques à chacun des environnements. L'idée de complémentarité et d'enrichissements réciproques entre contextes d'intervention et dispositifs de formation associés est ici défendue.

Le tutorat entre pairs : mises en œuvre et contextes d'intervention variés

Le tutorat entre pairs appartient à la famille des pratiques pédagogiques désignées sous le vocable d'apprentissage par les pairs et définies comme l'ensemble des stratégies d'acquisition de connaissances et d'habiletés au travers de l'aide active d'un alter-ego (Topping, 2005). L'examen de la littérature met en évidence des unités de fonctionnement (dyade ou groupe restreint) et des degrés de structuration (agencement des dyades/groupes, définition ou non de rôles particuliers) multiples au sein des pratiques d'apprentissage par les pairs.

S'agissant du tutorat entre pairs, la dyade représente l'unité de fonctionnement et les rôles de tuteur et de tutoré sont définis a priori. Cependant, l'expérimentateur ou l'enseignant 
« choisit » d'inclure l'ensemble ou une partie de la classe dans la stratégie de tutorat, de faire varier le degré de symétrie (compétence, âge, genre) entre les membres des dyades, et d'autoriser ou non l'alternance des rôles prescrits. Ces choix sont largement déterminés par les objectifs de recherche ou les enjeux éducatifs mais également par la nature du contexte d'intervention. Ainsi, le recours aux procédures de tutorat en dyades dissymétriques sans alternance des rôles (tutorat «fixe »), dans la littérature anglosaxonne, vise prioritairement le soutien d'élèves en fort échec scolaire ou présentant des déficiences mentales et/ou motrices dans une perspective d'intégration. Les études empiriques portant sur ces formes de tutorat s'inscrivent le plus souvent en contexte « expérimental ». À l'inverse, les procédures de tutorat en dyades symétriques avec alternance des rôles (tutorat "réciproque » et tutorat « en classe entière ») se déroulent fréquemment en contexte « écologique » de classe entière.

\section{La formation d'élèves tuteurs : condition essentielle d'efficacité du tutorat}

Parmi les facteurs d'efficacité des situations d'interaction de tutelle, la structuration des interactions a été explorée. Souvent décrite de manière allusive, elle nécessite des investigations supplémentaires. Dans le cas du tutorat entre pairs, ce travail s'organise notamment autour de la question de la formation ou de l'entraînement d'élèves tuteurs dans différents dispositifs. Les interrogations soulevées deviennent alors: suffit-il de mettre ensemble deux élèves de niveaux différents en prescrivant le rôle de tuteur au plus avancé pour qu'ils tirent profit l'un et/ou l'autre de l'interaction ? Peut-on se satisfaire de l'emploi du tutorat spontané entre élèves ou à l'inverse faut-il organiser en amont les interactions en formant les tuteurs? Les conclusions des recherches empiriques spécifiquement centrées sur cette problématique plaident majoritairement en faveur d'une nécessaire préparation de l'élève à l'exercice de la fonction de tuteur (Lafont \& Ensergueix, in press).

Conformément à la notion originelle de tutelle définie par Bruner (1983), le tuteur expert doit étayer l'activité du tutoré novice en apportant l'aide juste nécessaire (située à l'intérieur de la zone proximale de développement du novice, Vygotski, 1934/1985). Pour assurer pleinement les fonctions de guidage, le tuteur doit être sensible aux besoins d'informations du tutoré tout en vérifiant son niveau de compréhension, reconnaître le besoin de participation active et croissante au processus d'apprentissage de son partenaire, et enfin évaluer la capacité à apprendre propre à chaque novice. Or, l'adoption d'une telle conduite s'avère souvent trop exigeante pour les élèves et émerge rarement de manière spontanée, notamment chez le jeune enfant. Ce dernier, même expert dans l'activité, n'assume qu'incomplètement le rôle de tuteur (Winnykamen, 1996). Les 
compétences tutorielles sont différentes des compétences dans la tâche et doivent être appréhendées comme de véritables contenus d'enseignement.

Dans le domaine des disciplines académiques, la formation d'élèves tuteurs est déclinée selon plusieurs axes d'intervention dissociés ou complémentaires: l'amélioration des compétences dans la tâche, le développement d'aptitudes communicationnelles ou encore l'élévation des capacités de lecture des performances et d'analyse des besoins du tutoré. Toutefois, si la préparation ou la structuration en amont des séquences interactives sont le plus souvent mentionnées par les chercheurs, rares sont ceux qui les décrivent explicitement et concrètement. L'un des objectifs du présent article est de décrire et comparer les dispositifs de préparation au rôle de tuteur dans les deux contextes précités : un dispositif quasi expérimental et une mise en œuvre en classe entière au cours des séances d'un cycle ordinaire d'EPS.

Singularité du domaine de l'acquisition des habiletés motrices et dispositifs de formation en contexte expérimental

L'intervention de guidage dans le domaine de la motricité est spécifique : "Développer la sensibilité des tuteurs aux besoins des tutorés pose de réelles difficultés dans le cas des habiletés motrices complexes. Ainsi, les relations but/moyens se révèlent parfois difficiles à conscientiser (...) la performance motrice est éphémère, donc pose des problèmes de trace en mémoire pendant les interactions de guidage, contrairement à des résolutions de problèmes logiques ou mathématiques pour lesquels les états successifs de résolution peuvent continuer à être visualisés par le tuteur comme par le tutoré. " (Lafont et al., 2005). Par conséquent, si les préparations au tutorat entre pairs dans le domaine des habiletés motrices suivent en partie les recommandations énoncées au paragraphe précédent, elles témoignent cependant d'une singularité notable.

Les travaux conduits dans l'enseignement secondaire par l'équipe VSTII (Laboratoire LACES 4140, Université Bordeaux 2) illustrent la transposition aux cas de diverses habiletés motrices complexes des principes élaborés par Lorence (2001) pour la résolution d'une tâche logico-mathématique. Les chercheurs proposent un cadre général de formation selon quatre registres : la tâche, les relations entre le tutoré et la tâche, les interactions de guidage, et les mises en situation de simulation de tutorat. Ils insistent également quant à la nécessité d'ajuster ces aspects génériques en fonction du type de procédure de guidage utilisé (imitation modélisation interactive, tutorat fixe, ou tutorat réciproque) et de la nature des habiletés sollicitées par la tâche motrice (morphocinétiques, téléocinétiques, ou mixtes). Récemment, Cicéro et Lafont (2007) ont confirmé l'intérêt du cadre général de formation précédemment cité dans le cas d'une 
procédure expérimentale de tutorat fixe en gymnastique avec des élèves de $5^{\mathrm{e}}$. Les tutorés ont manifesté des progrès moteurs plus élevés quand leur tuteur était formé car ce dernier leur permettait de tirer un plus grand profit des aides fournies. Les tuteurs formés, comparés aux tuteurs spontanés, ont fait appel à des informations plus variées et mieux ancrées dans la réalisation antérieure du tutoré.

Par ailleurs, deux études fournissent des exemples de dispositifs de formation d'élèves tuteurs mise en œuvre ailleurs que dans l'enseignement secondaire. D'une part, Legrain, Arripe (d'), et Gernigon (2003) se sont employés à former des étudiants au managérat d'un pair dans le cadre d'un cycle de boxe française en STAPS. Pour ce public novice de jeunes adultes, les auteurs ont modélisé un dispositif de formation extrêmement structuré et détaillé (cf. $1^{\mathrm{e}}$ étude de cet article). D'autre part, Houston-Wilson et al. (1997) se sont intéressés à la question de la préparation d'élèves de primaire au tutorat de pairs présentant des déficiences mentales. Ici, les tuteurs étaient d'abord recrutés pour leurs prédispositions à exercer leur fonction (comportement exemplaire en classe, niveau élevé de compétence dans la tâche motrice, fort désir de participer à l'étude) puis entraînés individuellement par un expérimentateur au cours de deux séquences de 30 min en amont de l'intervention. L'entraînement comportait des simulations de tutorat visant à stimuler chez le tuteur la formulation de feedbacks positifs généraux et spécifiques et le déclenchement opportun des actions du tutoré. Chacun des tuteurs devait atteindre un critère de réussite fixé par l'expérimentateur avant d'endosser son rôle en situation réelle.

Ces dispositifs ont tous été pensés pour répondre aux contraintes d'un contexte « expérimental » uniquement. La mise en œuvre de la formation était systématiquement assurée par l'expérimentateur, auprès d'un public restreint d'élèves sélectionnés, et dans un espace (spatial et temporel) spécifique. Or, on assiste dans une période assez récente à des changements de paradigmes dans plusieurs domaines liés à l'intervention. En premier lieu, certains chercheurs, à la suite de Vygotski, témoins d'héritages plus ou moins directs ont mis en évidence le rôle du contexte de l'interaction (Cole et Engestrom, 1993) et des partenaires (Wertsch, 1985). Dans le champ de la didactique des disciplines, émerge l'importance du milieu didactique ou encore de l'agencement des tâches. Ainsi, par exemple, le milieu didactique constitue un modèle dialogique et collaboratif, c'est la partie de la situation d'enseignement avec laquelle l'élève est mis en interaction. II est défini par des aspects matériels (instruments, documents, organisation spatiale...) et la dimension sémiotique associée (que faire avec, pourquoi faire avec, comment faire avec...). Par ailleurs, dans le domaine de l'intervention pédagogique selon le point de vue de l'action située, les recherches actuelles mettent en exergue le couplage de l'action et 
du contexte (Durand, 2001). Ainsi, la communication et les évènements qui surviennent à l'intérieur de la classe (relations enseignant enseigné ou entre élèves) sont inscrits et enchâssés dans un contexte qui assume un rôle fort. Pour ces raisons, il paraissait essentiel de valider un dispositif explicite de formation d'élèves tuteurs à la fois en contexte expérimental et en contexte de classe entière pour une même activité physique et sportive (APS).

Objectif, cadre général de recherche, et plan du présent article

L'objectif de cette recherche est de montrer l'intérêt d'étudier une même procédure de tutorat (ici de tutorat réciproque) dans deux contextes d'intervention distincts.

Le cadre général de recherche est le suivant:

- La procédure est le tutorat réciproque entre pairs

- L'APS support est le tennis de table

- Le niveau d'enseignement est le secondaire $\left(2^{\mathrm{e}}\right.$ cycle $)$

- L’implantation géographique de chacun des deux collèges est urbaine

- L'origine socio-économique du public scolaire est homogène (moyenne à favorisée)

La recherche se décline en deux études : l'une conduite en contexte expérimental, l'autre en contexte de classe entière. Chacune des études est successivement présentée selon (a) le contexte d'intervention, (b) le dispositif de formation, (c) les principaux résultats. Puis, un tableau synthèse comparant les deux études est proposé. Enfin, l'idée de complémentarité et d'enrichissements réciproques entre contextes d'intervention et dispositifs de formation associés est discutée en conclusion.

\section{Première étude}

1. 1. Contexte d'intervention expérimental

Sélection des participants et constitution de groupes expérimentaux

L'ensemble des élèves de sept classes de $3 e(N=182)$ a été prétesté dans la tâche motrice expérimentale en amont de l'intervention. L'objectif était de sélectionner les participants présentant un niveau initial d'habileté allant de «débutant » à « intermédiaire ». À l'issue du pré-test, 144 élèves (72G/72F ; $M=15,5$ ans, +/- 1,1) étaient retenus pour participer à la 1e étude. En fonction du niveau initial d'habileté et du genre des participants, six groupes expérimentaux $(n=24)$ ont été formés (tutorat formé mixte ou non mixte, tutorat spontané mixte ou non mixte, pratique physique individuelle formée ou spontanée). Les élèves placés en dyades dans les conditions de tutorat affichaient un niveau similaire d'habileté dans la tâche-cible. 
Tâche motrice expérimentale et spécifique

La tâche-cible était de nature perceptivo-motrice et décisionnelle. Un adulte expert envoyait 20 balles sur la demi-table du participant qui devait réagir et renvoyer la balle après s'être déplacé. Le joueur disposait de 2 essais entrecoupés d'un temps-mort (1min). L'enregistrement vidéo était assuré par une caméra numérique fixée sur un trépied.

Mesures dépendantes d'ordre moteur, cognitif, et motivationnel

L'étude visait à confirmer les bénéfices comportementaux chez les élèves d'une formation au tutorat réciproque et à étendre ces bénéfices aux registres de la cognition et de la motivation. Les mesures motrices concernaient l'efficacité de l'action et la pertinence du choix (Ensergueix et Lafont, 2007). Les mesures cognitives incluaient les connaissances déclaratives, les compétences managériales en situations virtuelle et réelle et le sentiment d'efficacité personnelle. Enfin, la mesure de la motivation était relative au sentiment d'autodétermination.

Procédure expérimentale " classique »

L'étude s'est déroulée au sein du cycle de tennis de table des 7 classes de $3^{\mathrm{e}}$ ( 7 séances de $2 \mathrm{~h}$ ) selon un plan expérimental « classique » en trois étapes (Ensergueix et Lafont, 2007). Durant tout le cycle, l'expérimentateur prenait en charge, dans un espace spécifique et isolé, les participants par vagues successives de quatre tandis que le professeur poursuivait son cours avec le reste de la classe.

(1) Le pré-test s'est effectué à la $1^{\mathrm{e}}$ séance, suite à la présentation de la tâche-cible et des différents questionnaires (cf. Mesures) par les expérimentateurs à l'ensemble de la classe. Les élèves rejoignaient individuellement la table expérimentale pour, d'une part, remplir les questionnaires et, d'autre part, réaliser la tâche-cible. Pour chaque classe, les participants étaient classés en ordre croissant de performance. Ce classement servait de référence pour former les six groupes expérimentaux.

(2) Le traitement comprenait la séquence de formation offerte entre la $1^{\mathrm{e}}$ et la $2^{\mathrm{e}}$ séance uniquement aux participants des trois conditions formées et l'entraînement dans la tâche-cible. À chacune des séances 2 à 5 , les participants réalisaient une fois la tâchecible. Aléatoirement et successivement, quatre sujets d'un même groupe expérimental étaient appelés à la table expérimentale. Les participants des groupes de pratique physique individuelle effectuaient seul la tâche-cible tandis que les trois autres ramassaient les balles. Ceux des groupes de tutorat étaient, en dyade, impliqués tour à tour dans la tâche-cible. À la $2^{\mathrm{e}}$ séance, ces modalités de pratique étaient formulées ainsi :

«Dans la séquence suivante, vous serez alternativement joueur et manageur. Le joueur réalise son $1^{\text {er }}$ essai pendant que le manageur l'observe en silence. Pendant le temps-mort, le manageur est libre de 
donner des conseils au joueur afin qu'il améliore sa performance à l'essai suivant. Le joueur réalise son $2^{e}$ essai pendant que le manageur l'observe en silence. À la fin de cette séquence, les rôles seront inversés ».

(3) Les post-tests immédiat et différé se sont déroulés respectivement aux séances 6 et 7 (espacées d'un délai de deux semaines). Ils incluaient, d'une part, la passation des différents questionnaires dans des conditions identiques au pré-test et, d'autre part, l'évaluation des compétences motrices et managériales en situation réelle selon les modalités décrites à la $2^{\mathrm{e}}$ séance. Par ailleurs, au post-test immédiat, les participants devaient analyser par écrit une séquence vidéo de la tâche-cible exécutée par un élève de $3^{\mathrm{e}}$ débutant et anonyme (évaluation des compétences managériales en situation virtuelle).

1. 2. Dispositif de formation en contexte expérimental

Le dispositif de formation de la $1^{\mathrm{e}}$ étude résultait de l'adaptation au cas du tutorat réciproque du cadre général de formation proposée par l'équipe de recherche VSTII pour des procédures de tutorat fixe (voir Annexe A pour une synthèse).

Objectif : identifier les cinq règles d'or du manageur

L'objectif de la formation était la prise de conscience des participants des comportements particulièrement attendus chez le manageur (Tableau 1). Ces derniers correspondent aux fonctions clés du tuteur initialement définies par Bruner (1983) et validées dans le cas de l'acquisition des habiletés motrices complexes par Lafont (1997).

Tableau I. Les cinq règles d'or du manageur

Règle 1 Observe attentivement ce que fait ton partenaire

Règle 2 Rappelle et démontre à ton partenaire ce qu'il faut faire

Règle 3 Laisse du temps à ton partenaire pour te poser des questions

Règle 4 Réconforte et encourage ton partenaire en difficulté

Règle 5 Félicite ton partenaire en réussite

La question centrale de la $1^{\mathrm{e}}$ séquence de TGN (cf. Mise en œuvre) concernait la $1^{\mathrm{e}}$ règle d'or du manageur. Elle était inscrite au tableau en ces termes : "Que doit faire le manageur pendant le jeu ? », accompagnée de la réponse : "Observer attentivement ». Les élèves étaient ainsi invités à : «Identifier 1 à 3 des défauts majeurs $d u$ joueur » et à «Proposer un conseil pour chaque défaut identifié » dans trois cas concrets.

La question centrale de la $2^{\mathrm{e}}$ séquence visait l'identification des quatre autres règles d'or du manageur. Sa formulation au tableau était : "Que doit faire le manageur pendant le temps-mort ? ». Les consignes suivantes étaient formulées : "Identifier 1 à 3 des défauts 
majeurs du manageur » et "Repérer 1 à 3 des principales qualités du manageur » pour trois cas concrets.

\section{Mise en œuvre à l'aide de la technique du groupe nominal}

Les élèves des groupes expérimentaux avec formation $(N=72)$ ont été répartis en 5 sousgroupes ( $n=12$ à 16). La mise en œuvre de la formation était identique pour chaque groupe, seul le créneau horaire différait. La formation se déroulait en dehors des cours d'EPS, entre $48 \mathrm{~h}$ après la $1^{\mathrm{e}}$ séance et $48 \mathrm{~h}$ avant la $2^{\mathrm{e}}$ séance, et sur deux périodes de classe de 55min. Elle mobilisait une salle annexe, une table de tennis de table, un tableau à roulettes, des feuilles vierges et des crayons. Un expérimentateur et trois élèves « complices » aux caractéristiques proches des participants étaient également impliqués. Par ailleurs, la formation dispensait les élèves de toute pratique physique et de tout exercice de la fonction de tuteur afin d'assurer la validité interne de l'étude. Ici, le défi majeur était donc d'assurer l'implication active des élèves uniquement par un travail d'observation. Ce défi a été en grande partie relevé par le recours à la Technique du Groupe Nominal (TGN).

La TGN est une méthode d'entretien où chacun des membres d'un groupe formule un avis, par écrit puis verbalement, à propos d'une question ou d'une situation (Delbecq, Van De Ven, et Gustafson, 1975). Pour cette $1^{\mathrm{e}}$ étude, la TGN visait à stimuler la réflexion des élèves relativement à deux questions centrales et était donc divisée en deux séquences de 40min. En l'absence de certains concepts clés ou en présence d'une hiérarchisation peu opérante, l'expérimentateur posait de courtes questions visant à solliciter la réflexion des participants. Cependant, à aucun moment, il ne fournissait directement les réponses aux questions. Avant de clore le protocole, l'expérimentateur s'assurait que les propositions finalement retenues par le groupe étaient comprises par tous.

1. 3. Principaux résultats de la 1e étude Formation indispensable à l'efficacité du tutorat réciproque

Conformément aux attentes, les participants formés au tutorat réciproque ont manifesté des bénéfices moteurs, cognitifs, et motivationnels supérieurs à ceux obtenus par les participants des conditions de tutorat spontané et de pratique physique individuelle. En revanche, aucune différence n'est constatée entre ces deux conditions. Ainsi, le dispositif de formation s'est avéré une condition, non pas favorable, mais indispensable à l'efficacité du tutorat réciproque en contexte expérimental. 


\section{Deuxième étude}

2. 1. Contexte d'intervention en classe entière Implication de l'ensemble élèves/enseignants et absence de mixité dans les dyades Tous les élèves $(N=113)$ de quatre classes de $4^{\mathrm{e}}$ ont été impliqués dans la $2^{\mathrm{e}}$ étude. II s'agissait de leur premier cycle de tennis de table au collège. Deux classes étaient impliquées dans une procédure de tutorat avec formation (Tutorat Formé, TF) tandis que les deux autres étaient engagées dans une procédure de tutorat réciproque sans formation (Tutorat Spontané, TS). Les dyades étaient symétriques (genre et compétence). Eu égard aux contraintes fortes du contexte écologique de la classe (absence, inaptitude...), le traitement des données ne concernait finalement que 92 élèves (46G/46F ; $M=14,2$ ans, +/- 1,2). Par ailleurs, les deux enseignants d'EPS, en responsabilité chacun de deux classes, étaient inclus dans l'étude. Ils conduisaient pour une classe une procédure TF et pour l'autre une procédure TS. Un guide complet de mise en œuvre des deux procédures et des séquences de formation avec un chercheur (comprenant des démonstrations/simulations des étapes clés de la séance à venir) étaient offerts aux enseignants.

\section{Situations d'apprentissage coopératives et compétitives variées}

À chaque séance, les élèves vivaient avec leur partenaire deux types de situation d'apprentissage conçues par le chercheur en accord avec le projet pédagogique et les habitudes de travail des enseignants (synthèse entre les préparations des enseignants et les choix du chercheur). D'une part, les élèves s'échauffaient en partenariat dans diverses épreuves, et, d'autre part, ils se " coachaient " alternativement lors de matchs individuels à thème. Chaque match comportait 2 sets de 3 min entrecoupés d'un temps mort de 2 min. Le coaching prenait place au temps mort. Chacune des situations d'apprentissage avait pour support des fiches de travail/score particulières. Enfin, trois caméras numériques placées sur trépieds assuraient l'enregistrement vidéo des comportements de « dyadescibles » (échantillon représentatif de 12 élèves par classe).

Mesures dépendantes, contrôle de la mise en œuvre, et entretiens Les mesures dépendantes étaient d'ordre moteur (scores aux épreuves en partenariat et analyse vidéo des performances en match), cognitif et motivationnel (variables identiques à la $1^{\mathrm{e}}$ étude). De plus, les élèves étaient invités à remplir des questionnaires de satisfaction à l'égard des rôles de coach/joueur et de la procédure de tutorat réciproque. Par ailleurs, le contrôle de la mise en œuvre des enseignants des procédures TF et TS était assuré par un expérimentateur à l'aide de divers « check-list » (Sherman, 1982). 
Plusieurs entretiens (d'autoconfrontation et semi-dirigé) ont également été conduits avec les enseignants.

\section{Procédure intégrée au déroulement normal du cycle}

Le cycle de tennis de table durait 10 séances. Les contenus d'enseignement étaient identiques pour les deux procédures et les élèves d'une même classe participaient ensemble aux différentes situations d'apprentissage. Toutefois, aux séances 3 à 9 et pendant 20min, les activités offertes aux élèves différaient : préparation aux rôles de coach/joueur pour les classes TF et matchs individuels libres pour les classes TS. Ainsi, les séquences de formation étaient remplacées par de la pratique en compétition dans les classes "contrôles ». Par ailleurs, en amont et pendant le cycle, plusieurs rencontres avec les enseignants ont été assurées par les chercheurs. L'objectif était double : d'une part, former à la mise en œuvre des procédures de tutorat et notamment à la conduite des séquences de préparation des élèves, et, d'autre part, recueillir leurs analyses et sentiments à l'égard de l'étude.

2. 2. Dispositif de formation en contexte de classe entière

Le dispositif de formation de la $2^{\mathrm{e}}$ étude correspondait à la transposition au domaine des habiletés motrices des principes élaborés par King (1997) pour une procédure de tutorat réciproque en classe de biologie humaine.

Objectif : identifier les clés d'une interaction réussie

L'objectif de la formation était d'identifier les étapes à suivre et les comportements à adopter par les deux partenaires pour réussir leurs matchs en équipe. À la séance 3 , les élèves étaient d'abord sensibilisés à l'importance égale des deux rôles joueur/coach, à la fois pendant le jeu et pendant le temps-mort. Le mot-clé «interaction » et ses conséquences (respect, écoute, réciprocité) étaient clairement définis et illustrés. Ils réfléchissaient ensuite au "pourquoi » et au "comment » des étapes de la fiche de formation. À la séance 4, l'enseignant commençait par souligner l'importance pour le coach d'envisager toujours son rôle au regard du thème de la séance et poursuivait en proposant aux élèves une démarche identique au $2^{\mathrm{e}}$ temps de la formation de la séance 3. À chacune des séances 5,6 , et 7 , la formation visait à identifier les critères de réussite propres au thème de la séance (placement, vitesse, service) et à renforcer l'appropriation des élèves de la fiche présentée à la séance 3 . Enfin, à la séance 9 , l'objectif de la formation était simplement de « rafraîchir les mémoires » après quinze jours de vacances. Mise en œuvre en EPS et à l'aide de la technique du brainstorming

La formation était assurée par les enseignants pour tous les élèves de la classe, collectivement et à plusieurs moments au cours du cycle. 
Le cœur du dispositif prenait place aux séances 3 et 4. L'enseignant demandait aux élèves de s'asseoir en demi-cercle devant un tableau aimanté et entamait une courte discussion visant à susciter l'intérêt des élèves. Puis, il affichait au tableau une fiche dont l'ensemble des mots-clés était masqué. Commençait alors une séquence de brainstorming (Osborn, 1959) ou «tempête d'idées » (12min). Le brainstorming est une technique de gestion des groupes qui stimule la production d'idées selon deux modes de pensée : divergente (créative) et convergente (critique). Ici, l'idée était d'impliquer un maximum d'élèves dans la recherche active des mots-clés masqués et de leurs synonymes. Les verbalisations/démonstrations de l'enseignant et les illustrations sur la feuille guidaient les élèves. Progressivement, l'enseignant dévoilait et discutait les motsclés. Avant de lancer le $1^{\text {er }}$ match en équipe, II signalait la présence d'un exemplaire de la fiche de formation à chaque table. Entre le $1^{\text {er }}$ et le $2^{\mathrm{e}}$ match, l'enseignant effectuait un « retour au calme ». Enfin, à la $3^{\mathrm{e}}$ séance uniquement, il contrôlait la mémorisation des élèves des mots-clés de la formation en leur demandant de compléter seul un exemplaire « à trous » de la fiche.

Aux séances 5,6 et 7 , la formation était déclinée en 2 séquences de 10min (respectivement en amont et en aval du $1^{\mathrm{er}}$ match). Deux dyades ( $\mathrm{A}$ et $\mathrm{B}$ ) réalisaient une simulation d'un match à thème tandis que le reste de la classe se répartissait de chaque côté de la table en deux groupes ( $A$ et $B$ ). Après une observation et/ou une écoute silencieuse, les groupes d'élèves donnaient successivement leurs impressions au regard d'une des dyades. Puis, l'enseignant explicitait et illustrait les propos formulés.

Enfin, à la séance 9, la formation comprenait à nouveau deux séquences : simulation de l'enseignant dans le rôle de coach avec un élève et simulation assurée uniquement par des élèves.

2. 3. Principaux résultats de la $2 e$ étude

\section{Supériorité du tutorat réciproque formé par rapport au tutorat réciproque spontané}

Conformément aux attentes, les élèves des classes où les enseignants avaient dispensé une formation au tutorat réciproque, ont obtenu des performances motrices et cognitives plus élevées que celles affichées par les élèves des classes où les enseignants avaient remplacé la formation par des séquences de jeu libre individuel. Ainsi, le dispositif de formation, bien que réduisant le temps de pratique physique effective des élèves, s'est révélé bénéfique en contexte de classe entière en EPS. Par ailleurs, les élèves " formés » ont manifesté une satisfaction supérieure à l'égard de leur partenaire et identique à l'égard du cycle complet en comparaison avec les élèves «spontanés ». Le dispositif de 
formation n'a donc pas été vécu par les élèves comme une contrainte mais plutôt comme une aide.

Tableau II Synthèse comparant les contextes d'intervention, dispositifs de formation, et principaux résultats des deux études

\begin{tabular}{|c|c|c|}
\hline & $1^{\mathrm{e}}$ étude & $2^{\mathrm{e}}$ étude \\
\hline $\begin{array}{l}\text { Contexte } \\
\text { d'intervention }\end{array}$ & Expérimental & Classe entière \\
\hline $\begin{array}{l}\text { Objectif(s) de } \\
\text { l'étude }\end{array}$ & $\begin{array}{l}\text { 1. Comparer les bénéfices } \\
\text { moteurs/cognitifs/motivationnels de } 3 \\
\text { conditions (TRP formé, TRP spontané, } \\
\text { Pratique individuelle) }\end{array}$ & $\begin{array}{l}\text { 1. Comparer les effets chez les élèves et } \\
\text { chez les enseignants de deux procédures } \\
\text { TRP (formé et spontané) en EPS } \\
\text { 2. Contrôler la mise en œuvre } \\
\text { élèves/enseignants des } 2 \text { procédures }\end{array}$ \\
\hline Hypothèse(s) & $\begin{array}{l}\text { 1. TRP formé > TRP spontané > Pratique } \\
\text { individuelle }\end{array}$ & $\begin{array}{l}\text { 1. TRP formé > TRP spontané ; } 2 \text {. } \\
\text { Exploratoire }\end{array}$ \\
\hline Participants & $\begin{array}{l}144 \text { élèves débutants sélectionnés a.p. de } \\
7 \text { classes de } 3^{\mathrm{e}}\end{array}$ & $\begin{array}{l}\text { Ensemble des élèves (113) et enseignants } \\
\text { (2) de } 4 \text { classes de } 4\end{array}$ \\
\hline Tâche(s) & $\begin{array}{l}\text { Situation perceptivo-motrice spécifique } \\
\text { face à un adulte expert et à une table } \\
\text { expé. isolée du cours ; passation alternée } \\
\text { des élèves }\end{array}$ & $\begin{array}{l}\text { Situations coopératives/compétitives } \\
\text { variées, en accord avec le projet } \\
\text { pédagogique et les habitudes de travail des } \\
\text { enseignants. }\end{array}$ \\
\hline $\begin{array}{l}\text { Recueil des } \\
\text { données }\end{array}$ & $\begin{array}{l}\text { Efficacité d'action et pertinence du choix; } \\
\text { connais. déclaratives, comp. } \\
\text { managériales virtuelles et réelles, SEP, } \\
\text { autodétermination }\end{array}$ & $\begin{array}{l}\text { Élèves : } \neq \text { mesures dép. (étude } 1 \text { ); } \\
\text { satisfaction / rôles et procédure } \\
\text { Enseignants : contrôle mise en œuvre; } \\
\text { entretiens autoconfrontation }\end{array}$ \\
\hline Procédure & $\begin{array}{l}\text { Protocole expé. "classique " en } \\
\text { périphérie des activités de la classe : pré- } \\
\text { test ( } \mathrm{S} 1: \text { sélection des participants) / } \\
\text { traitement (S2 à S5: entraînement ds la } \\
\text { tâche expé) / post-tests ( } 6 \text { et } \mathrm{S} 7 \text { ) }\end{array}$ & $\begin{array}{l}\text { Procédure intégrée au déroulement normal } \\
\text { du cycle ; contenus et déroulement des } \\
\text { séances identiques pour les } 4 \text { classes } \\
\text { excepté le tps alloué à la formation } \\
\text { remplacé par un tps de jeu libre }\end{array}$ \\
\hline $\begin{array}{l}\text { Dispositif de } \\
\text { formation }\end{array}$ & $\begin{array}{l}\text { Transposition au cas du TRP du cadre } \\
\text { général proposé par l'équipe de } \\
\text { recherche VSTII pour des procédures de } \\
\text { tutorat fixe }\end{array}$ & $\begin{array}{l}\text { Transposition au domaine des habiletés } \\
\text { motrices des principes élaborés par King } \\
\text { (1997) pour du TRP en classe de biologie }\end{array}$ \\
\hline Objectif(s) & $\begin{array}{l}\text { Identifier «Les cinq règles d'or du } \\
\text { manageur "; } 2 \text { points majeurs } \\
\text { - Évaluer la perf. du joueur et fournir des } \\
\text { conseils ds un tps réduit } \\
\text { - Manifester des attitudes pro-sociales } \\
\text { (encourager, féliciter...) }\end{array}$ & $\begin{array}{l}\text { Identifier «Les clés d'une interaction } \\
\text { réussie »; } 2 \text { points majeurs : } \\
\text { - Importance = des rôles joueur/coach pdt } \\
\text { le match et le tps-mort } \\
\text { - Adapter coaching selon thème de la } \\
\text { séance et besoins du joueur }\end{array}$ \\
\hline $\begin{array}{l}\text { Mise } \\
\text { oeuvre }\end{array}$ & $\begin{array}{l}\text { Hors EPS (entre } \mathrm{S} 1 \text { et } \mathrm{S} 2) \text {, par un } \\
\text { expérimentateur, en petits groupes }(n= \\
12 \text { à 16), sur } 1 \text { séquence de } 2 \mathrm{~h} \text {, } \\
\text { technique du groupe nominal: } \\
\text { simulations par un expert et } 3 \text { élèves } \\
\text { complices }\end{array}$ & $\begin{array}{l}\text { En EPS (S3 à S9), par l'enseignant (formé } \\
\text { en amont), en classe entière, sur plusieurs } \\
\text { séquences ( } 15 \text { à } 25 \mathrm{~min} / \text { séance), } \\
\text { technique du brainstorming: simulations } \\
\text { par l'enseignant et les élèves }\end{array}$ \\
\hline $\begin{array}{l}\text { Principaux } \\
\text { résultats }\end{array}$ & $\begin{array}{l}\text { 1. TRP formé }>\text { TRP spontané = Pratique } \\
\text { individuelle }\end{array}$ & $\begin{array}{l}\text { 1. TRP formé }>\text { TRP spontané } \\
\text { 2. Mise en œuvre efficace } \\
\text { élèves/enseignants des } 2 \text { procédures }\end{array}$ \\
\hline
\end{tabular}

Note $:$ TRP $=$ Tutorat Réciproque entre Pair

\section{Mise en œuvre efficace des enseignants et des élèves du tutorat réciproque}

De manière originale, la $2^{\mathrm{e}}$ étude contrôlait la mise en œuvre des deux procédures de tutorat par les enseignants et les élèves. Une attention particulière était accordée au dispositif de formation. L'analyse des résultats révèle une mise en œuvre conforme aux 
attentes proche de $90 \%$ pour les deux procédures. Concernant la formation, un pourcentage identique est constaté pour les deux enseignants. Ainsi, les deux procédures de tutorat réciproque et le dispositif de formation se sont avérés adaptés aux contraintes particulières d'un contexte de classe entière en EPS.

\section{Conclusion}

L'efficacité de deux dispositifs de formation d'élèves au tutorat réciproque, associés, pour l'un, à un contexte expérimental et, pour l'autre à un contexte de classe entière a été vérifiée. Par ailleurs, et de manière originale, la recherche atteste de la pertinence d'adopter parallèlement une démarche expérimentale et une perspective prenant en compte l'écologie de la classe pour l'examen d'un même objet d'étude. Ces démarches, reflétant chacune un grand courant de la recherche scientifique, sont trop souvent, à tort, juxtaposées ou encore opposées (Baudrit, 2000).

L'objectif général de la recherche était de tester l'efficacité et la faisabilité de dispositifs de formation au tutorat réciproque entre pairs d'élèves de classes de $4^{\mathrm{e}}$ et $3^{\mathrm{e}}$ pour l'acquisition d'habiletés motrices en tennis de table. En considérant deux contextes d'intervention distincts, nous avons pu répondre à cet objectif de plusieurs manières. En effet, chacun d'eux proposait des caractéristiques propices à l'étude de problématiques de recherche particulières.

Dans le cas du contexte expérimental : le dispositif et le questionnement étaient proches des travaux antérieurs relatifs au tutorat fixe d'où une centration sur la nature de la procédure et de l'APS. La présence de dyades symétriques avec alternance des rôles de tuteur / tutoré et la sollicitation d'habiletés stratégiques nous a conduit à réfléchir à un dispositif de formation de type "émergeant ». De plus, eu égard à la nature du protocole expérimental, il y avait nécessité de proposer une formation excluant une pratique physique additionnelle. Le recours à une technique émergeante d'entretien en groupe : la TGN a constitué notre réponse au plan de la méthode.

Dans le cas du contexte écologique de classe : les caractéristiques étaient éloignées des travaux antérieurs et le contexte spécifique. En conséquence, nous avons choisi de nous centrer sur trois éléments majeurs nouveaux et incontournables : la mise en œuvre de la formation par les enseignants eux-mêmes («novices » dans l'usage du tutorat réciproque), l'hétérogénéité du public d'élèves et des situations d'apprentissage et enfin des contraintes spatio-temporelles fortes. La formation en amont des formateurs, la création de supports didactiques nouveaux et évolutifs à partir des préparations des 
enseignants, l'emploi d'une technique d'entretien en groupe plus simple et souple ont constitué nos éléments de réponse à l'évaluation des conditions initiales.

En portant un regard croisé sur ces deux dispositifs, il est possible d'en éclairer les forces et faiblesses essentielles. D'une part, à l'inverse du dispositif de formation en contexte de classe entière, le dispositif expérimental n'offrait pas aux élèves la chance de vivre concrètement des simulations de tutorat et proposait une formation " massée » sur une séquence unique de deux heures. D'autre part, contrairement au dispositif de formation en contexte expérimental, le dispositif en classe entière nécessitait plusieurs séquences de formation des enseignants et nous a contraint à utiliser la méthode de " brainstorming » plutôt que l'entretien de groupe (TGN). Les caractéristiques propres à chaque contexte ont donc fortement conditionné l'élaboration de dispositifs de formations, la procédure d'investigation et au final, la nature et la quantité des données mesurées et recueillies. Par exemple la conception particulière de l'investigation en classe nous contraint à vérifier la validité de la mise en œuvre par les enseignants. Toutefois, nous plaidons ici plutôt en faveur d'une complémentarité entre les approches autour d'un objet et de questions de recherche communs. Ainsi, si l'emploi de la TGN, technique avérée efficace en contexte expérimental, semblait peu convenir au contexte de classe particulier à notre recherche, elle pourrait cependant s'envisager dans un autre contexte de classe. Par exemple au sein d'un cycle d'une APS en extérieur lors d'une séance de mauvais temps. La démarche expérimentale reste tout de même soucieuse d'une validité externe et propose par conséquent des pistes susceptibles d'être réinvesties en contexte ordinaire de classe. L'efficacité de la TGN a d'ailleurs été largement démontrée en milieu scolaire (MacPhail, 2001). Par ailleurs, la démarche de formation en direction des enseignants ordinaires, indispensable en contexte écologique, s'appuie en priorité sur l'expérience des chercheurs suite à la mise en œuvre de dispositifs expérimentaux utilisant une formation.

Enfin, les composantes de la formation en contexte écologique de classe ont bénéficié du regard plus rigoureux de la recherche expérimentale avec notamment la possibilité d'évaluer des variables socio-émotionnelles à l'aide de questionnaires spécifiques. Le contexte de classe favorise l'émergence de composantes nouvelles de formation dont la démarche expérimentale peut se charger ensuite d'optimiser. II s'agit véritablement d'un fonctionnement en " aller-retour » de type systémique et non d'un système unidirectionnel ou en « vase clos ». On retrouve ici l'idée défendue par Rohrbeck et al. (2003) invitant chercheurs et enseignants à travailler de concert pour améliorer l'efficacité et la faisabilité des stratégies d'apprentissage par les pairs.

Cet article témoigne de l'importance du contexte d'intervention dans l'exploration d'une 
perspective théorique (ici, le tutorat réciproque entre pairs). Cette variable clé pèse à la fois sur la procédure d'investigation, la nature et le mode de recueil des données et sur le type de formation des tuteurs mis en oeuvre. Ainsi, nous proposons des alternatives quant à la formation d'élèves tuteurs en EPS selon les contextes. Ce travail demande à être approfondi grâce à des investigations sur le même modèle, en variant par exemple le type de procédure de tutorat ou encore la durée de l'intervention (étude longitudinale).

\section{Bibliographie}

Baudrit, A. (2000). Le tutorat: un enjeu pour une pratique pédagogique devenue objet scientifique ? (Note de synthèse). Revue Française de Pédagogie, 132, 125-153.

Bruner, J.S. (1983). Le développement de l'enfant : savoir faire, savoir dire. Paris, PUF.

Cicero, C., \& Lafont, L. (2007). Interaction de tutelle et imitation modélisation interactive entre élèves : l'effet de la formation d'élèves tuteurs en gymnastique sportive. Bulletin de psychologie, 60, 335-348.

Cole, M., \& Engestrom, Y. (1993). A cultural-historical approach to distributed cognition. In G. Salomon (Eds.), Distributed cognitions: Psychological and educational considerations (pp.1-46). New York: Cambridge University Press.

Delbecq, A.,Van De Ven, A., \& Gustafson, D. (1975). Group techniques for program planning. Glenview, IL, Scott, Foresman and Company.

Durand, M. (2001). Chronomètre et survêtement. Paris : Editions Revue EP.

Ensergueix, P. \& Lafont, L. (2007). Formation au Managérat Réciproque en tennis de table chez des élèves de 14-15 ans : tentative de modélisation et mesure des effets. eJRIEPS, 12, 51-67.

Houston-Wilson, C., Dunn, J., van der Mars, H., \& McCubbin, J. (1997). The effect of peer tutors on motor performance in integrated physical education classes. Adapted Physical Activity Quarterly, 14, 298-313.

King, A. (1997). Ask to think-Tel why: A model of transactive peer tutoring for scaffolding higher level complex learning. Educational psychologist, 32, 221-235.

Lafont, L., \& Ensergueix, P. (in press). La question de la formation d'élèves tuteurs: considérations générales, application au cas des habiletés motrices. Carrefours de l'éducation.

Lafont, L. (1997). Interactions sociales et acquisition des habiletés motrices : modalités d'analyse, Actes du Congrès International ACAPS. Marseille : ACAPS. 
Lafont, L., Cicero, C., Vedel, A. \& Viala, M. (2005). Apports de la psychologie sociale à l'intervention en EPS : Rôle des interactions tutorielles et des « coping » modèles. eJRIEPS, 8, 89-102.

Legrain, P., Arripe-Longueville (d'), F., \& Gernignon, C. (2003). The influence of trained peer tutoring on tutors' motivation and performance in a French boxing setting. Journal of Sports Sciences, 21, 159-160.

Lorence, B. (2001). Le tutorat entre enfants. Compétence sociale et performance : Quelles incidences ? Actes du $4^{e}$ congrès international: Actualité de la recherche en éducation et formation, Lille3, [CD-Rom].

Osborn, A. (1959). L'imagination constructive, Dunod.

Rohrbeck, C., Ginsburg-Block M., Fantuzzo J., \& Miller T. (2003). Peer-assisted learning interventions with elementary school students: A meta-analytic review. Journal of Educational Psychology, 95, 240-257.

Sherman, M. (1982). Style analysis checklist for Mosston's spectrum of teaching styles. Unpublished manuscript, University of Pittsburgh.

Topping, K. J. (2005). Trends in peer learning. Educational Psychology, 25, 631-645.

Wertsch, J. (1985). Vygotsky and the social formation of mind. Cambridge: Harvard University Press.

Winnykamen, F. (1996). Expert et/ou tuteur : les comparaisons des dyades adulte/enfant et enfant/enfant peuvent-elles éclairer le processus de guidage ? Revue de Psychologie de l'Education, 2,13-35. 


\section{Annexe}

Annexe A

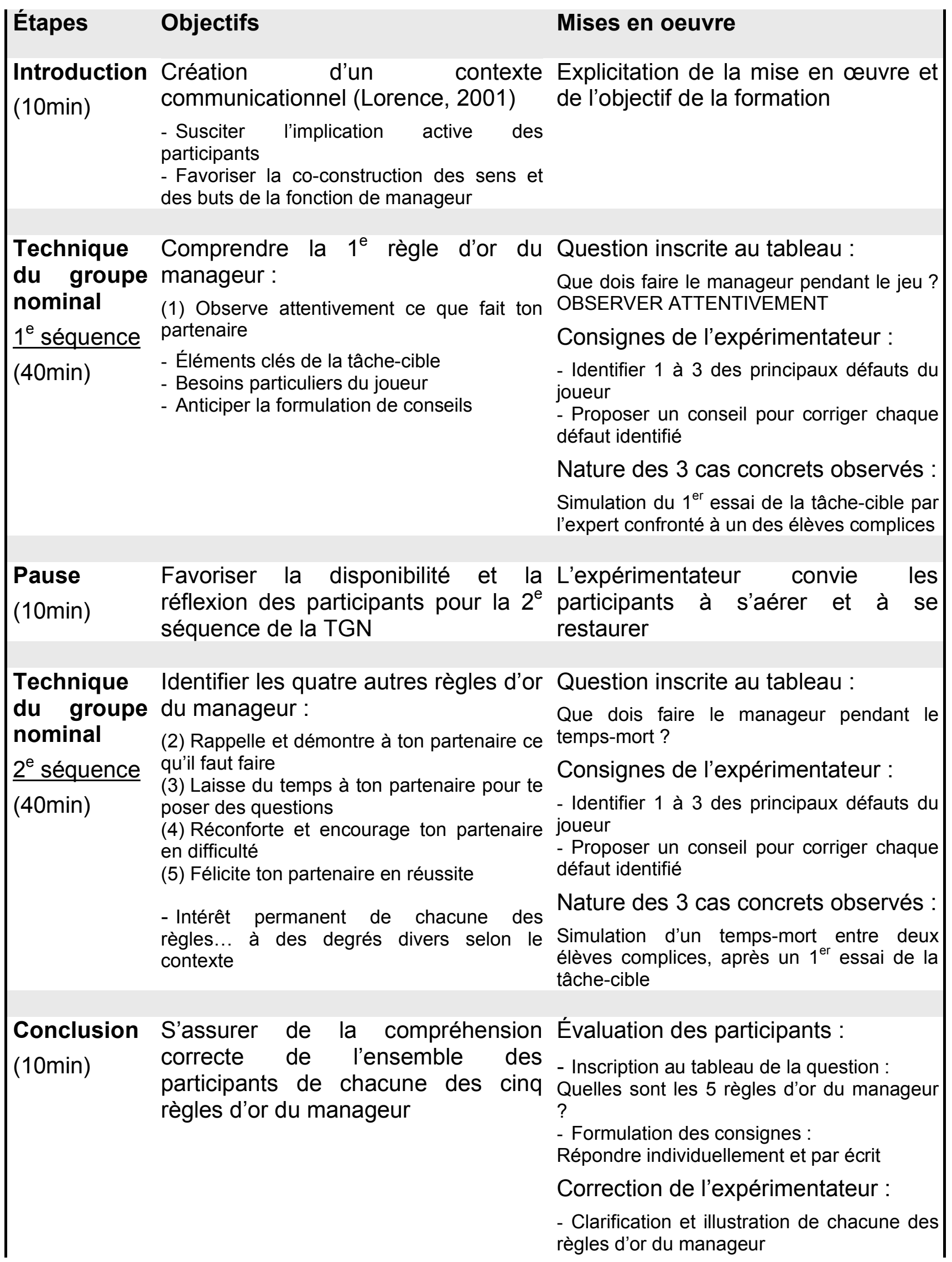

